\title{
Voltage sensors: challenging, but with potential
}

\author{
Nina Vogt \\ Genetically encoded voltage sensors are coming of age, but their use still poses challenges that must be \\ addressed from multiple angles.
}

Neurons process information with the help of electrical signals. Changes in electrical potential across neuronal membranes have been classically measured with electrodes, similar to the measurement of voltage in electrical circuits. Voltage sensors are exciting tools that allow us to instead visualize these voltage changes through a microscope lens. Nature Methods spoke with scientists who develop and use genetically encoded voltage sensors about the different tools available to the community, the challenges their developers face and considerations for their application.

When we think about membrane potentials, we think mostly about neurons. But "every cell has a membrane, and in principle this membrane can support voltage," says Adam Cohen, a Howard Hughes Medical Institute investigator at Harvard University. Examples of electrically active cells are cells in the heart, pancreas and muscle. Cohen has found that even bacteria can generate fast changes in membrane potential. The membrane potential is built and maintained by ion pumps and channels that actively or passively transport ions across the membrane.

However, as electrical activity is the currency of information in neurons, it is in these cells that measuring voltage changes is of particular interest. The interplay of activity in many neurons forms the biological basis for actions and emotions. Analysis of neuronal ensembles with voltage sensors can thus give us insight into how electrical signals contribute to higher order functions.

\section{Measuring voltage changes in biology}

Membrane potentials can be measured in a number of ways. Various electrophysiological approaches for intracellular and extracellular recording have been developed in the past century. "But there are some limitations to the number of electrodes you can stick into the tissue as compared to the number of cellsw which are around," cautions Thomas Knöpfel, who recently became the Chair of Optogenetics a n d Circuit Neuroscience at Imperial College in London. In general, these approaches have a relatively low throughput, meaning that only a few tens or hundreds of neurons can be analyzed per experiment. Intracellular recordings can be especially difficult (for example, when

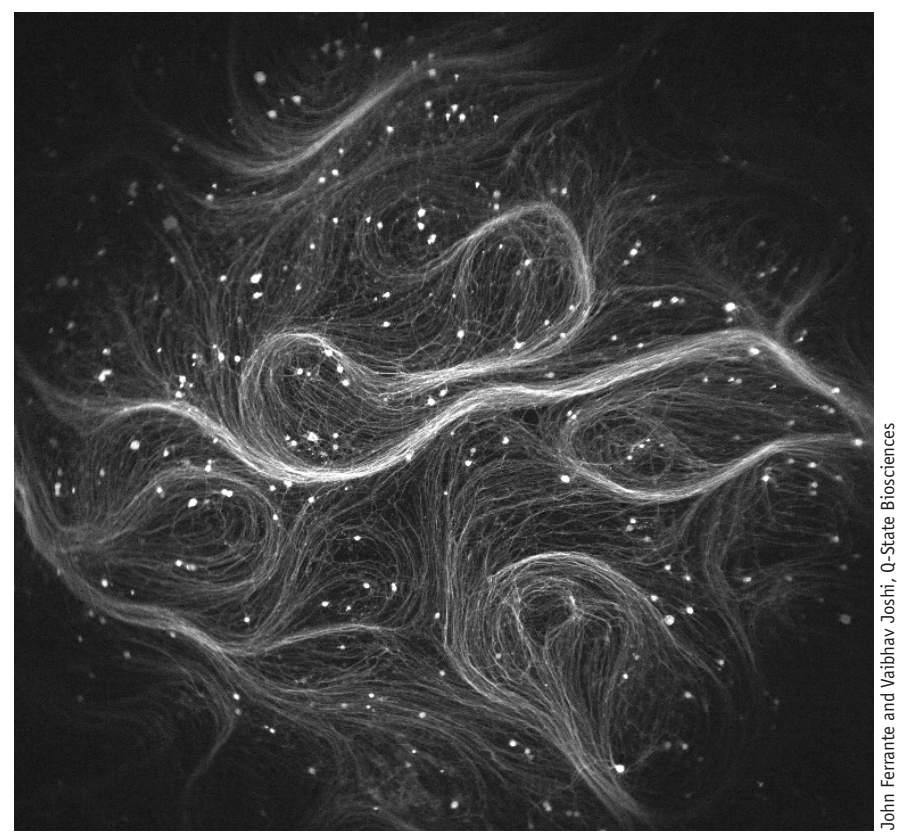

Rat dorsal root ganglion cells expressing 0ptopatch, a construct containing a QuasAr sensor. come through," explains Knöpfel. These dyes can still be useful when injected into single cells to avoid issues with background, but this requires access to single cells and reduces throughput.

Evan Miller and his team at the University of California at Berkeley have overcome the problem of indiscriminate labeling by developing a photoactivatable voltage-sensitive dye. Their SPOT2.1.Cl consists of the voltage-sensitive dye VoltageFluor2.1.Cl and a photosensitive caging group. The dye turns fluorescent upon uncaging and can be used to image voltage changes in defined neurons.

But most efforts at tool development are focused on genetically encoded voltage indicators, or GEVIs. "It's changing so fast that next month there is going to be a new 


\section{Voltage sensing domain-based VSFPs}

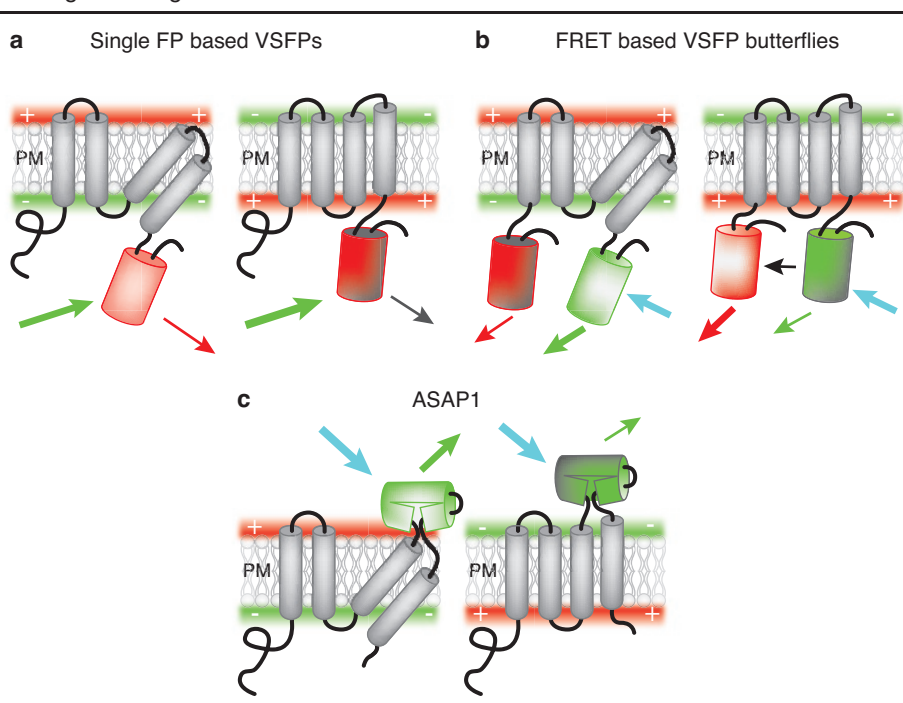

e

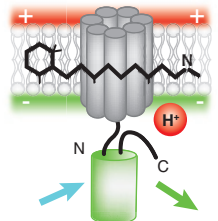

Opsin-based GEVI designs d Opsin-based voltage indicator Arch

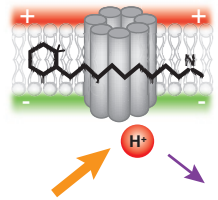

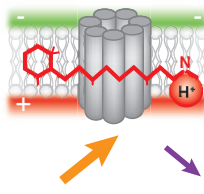

Schematic overview of voltage-sensor designs.

set of voltage indicators out there," says Cohen. Because these sensors are genetically encoded, they can be targeted to specific cell types, at least for cell types that are genetically addressable. Thus identifying the cells of interest is not a problem, and at the same time the throughput can be high.

\section{Genetically encoded voltage indicators}

In 1997, Ehud Isacoff and his team at the University of California at Berkeley reported the first genetically encoded voltage sensor, consisting of a fusion between a potassium channel and a fluorescent protein ${ }^{1}$. Voltagedependent changes in the channel resulted in fluorescence changes. Since then, several generations of voltage sensors have been developed. They fall into two classes: rhodopsin-based sensors, and sensors that are fusions between voltage-sensitive domains and fluorescent proteins, like the one developed by Isacoff.

For the sensors based on voltage-sensing domains, voltage changes can be visualized either by a single fluorescent protein or by a FRET (Förster resonance energy transfer) pair. The sensor VSFP-Butterfly, developed in Knöpfel's lab, is an example of the latter type ${ }^{2}$. In VSFP-Butterfly, the voltage-sensi- tive domain, obtained from a Ciona voltagesensitive phosphatase ( $C i$-VSP), is located between the two fluorescent proteins of the FRET pair, but sensors with fluorescent proteins at different locations have also been developed. Knöpfel has used the Butterfly sensor mainly for imaging electrical activity in populations of neurons. The popular sensor ArcLight from Vincent Pieribone's lab at Yale University is an example of a sensor in which voltage changes are reported by a single fluorescent protein ${ }^{3}$. ArcLight has been used for subthreshold and action-potential imaging in single cells in the Drosophila brain.

Another iteration of a sensor in which a voltage-sensitive domain is coupled to a single fluorescent protein is ASAP1, from Michael Lin and his team at Stanford University ${ }^{4}$. In this design, a circularly permuted fluorescent protein is inserted between two transmembrane helices in the voltage-sensitive domain of chicken VSP. "The idea is that a change in voltage moves one transmembrane helix relative to the others, and that sort of shears this protein and cracks it open and decreases its fluorescence," explains Cohen. He considers ASAP1 as the most sensitive and fastest member of the sensor class based on voltage-sensitive domains.
The opsin-based sensors are a more recent development, with the first example published by Cohen's group. These indicators are derived from the microbial proton pump Archaerhodopsin-3 (Arch) ${ }^{5}$. Though Arch and related sensors do report voltage changes with changes in fluorescence, "one major issue was that they were very dim," cautions Knöpfel. Furthermore, Arch is not an inert sensor; consistent with its original role, Arch pumps protons upon illumination with red light, thereby changing the membrane potential it reports.

Arch has since been mutated in different ways to improve baseline fluorescence, kinetics and dynamic range; to reduce the illumination intensity necessary to elicit a signal; and to diminish its proton-pumping activity. The most recent developments are QuasAr1 and -2 from Cohen's team ${ }^{6}$, which have enabled all-optical electrophysiology in the mouse, and the improved-fluorescence Archer1 and -2 (ref. 7), developed by Viviana Gradinaru and her group at the California Institute of Technology, which have been used to image neural activity in Caenorhabditis elegans.

Arch has also been mutated to act as a voltage integrator with memory function. Although still at the proof-of-principle stage, these sensors could potentially be useful for readouts of activity at a later time point, such as when combining functional analysis with morphological studies.

A strategy to increase the brightness of rhodopsin-based sensors is to combine them with fluorescent proteins to form FRET pairs. The fluorescent protein serves as the FRET donor while the opsin functions as both voltage sensor and FRET acceptor. Thus the fast voltage-sensing properties of the opsins are maintained while brightness is increased. The most recent members of this design family are MacQ-mCitrine and MacQ-mOrange2, which were developed by Mark Schnitzer and his team at Stanford

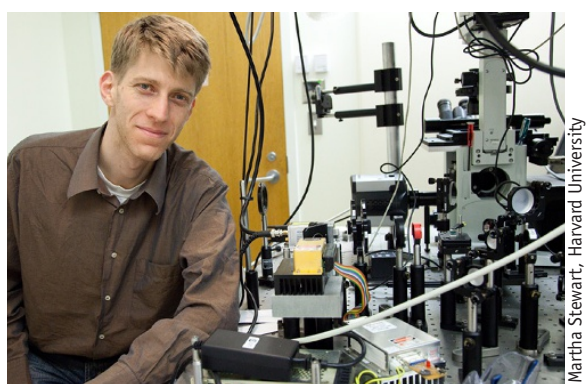

New voltage sensors are constantly coming out, says Adam Cohen. 
University $^{8}$. The researchers applied these tools to visualize voltage changes in Purkinje neurons in the cerebella of live mice.

\section{Voltage sensors at work}

With such a variety of genetically encoded voltage indicators, it might be difficult for someone new to the field to pick one. In fact, there might not be an ideal sensor. "It really depends on the experimental needs and preparations and the question that's being asked, labeling density and so on," offers

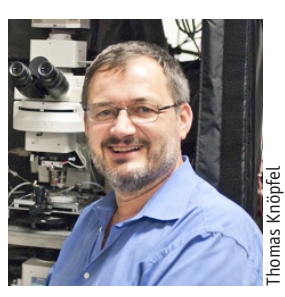

Voltage imaging can help researchers look at the bigger picture, says Thomas Knöpfel.
Schnitzer. He recommends choosing an indicator that is tailored to the experimental needs. "If you tell me what you are looking at are subthreshold potentials, I might devise it one way; if you are looking at spikes, I would devise it another way," says Schnitzer regarding the desired indicator properties.

What are the types of experiments voltage sensors can be useful for? "There are people who are interested in looking at large-scale circuit dynamics, recording from many neurons in parallel over a large field of view, possibly in three dimensions," says Cohen. Others may be interested in looking at synaptic transmission or at dendritic integration (how inputs get summed together to result in a decision about whether a cell fires), he says.

Although it is feasible to image the electrical activity of populations of neurons in vivo, it is technically challenging to image singlecell activity in this context. Questions at the scale of single-cell activity are therefore currently addressed mostly in neuronal cell culture. Knöpfel is an advocate of larger-scale in vivo voltage imaging for the study of populations of neurons. "My feeling is also that the domain where voltage imaging can really help is looking at the bigger picture, the integration of activity across cortical areas." But in mixed populations, voltage changes may cancel each other out when averaged across cell classes. The possibility of genetically targeting the voltage indicators to defined cell populations-for example, subsets of inhibitor interneurons-is therefore essential, Knöpfel says.

In vivo imaging of electrical activity in single cells has been demonstrated in smaller model organisms. In Drosophila, for example,
ArcLight has provided insight into neural communication in circadian clock neurons, and voltage changes in response to odor stimuli have been visualized in C. elegans sensory neurons. In the mouse brain, however, voltage imaging at the single-cell level is currently not yet practical, according to Cohen.

\section{The difficulties}

Why is voltage imaging so technically challenging? It is mainly because there are typically a limited number of sensor molecules to record from and because a high imaging speed is needed to capture this fast process. Together, these two factors result in a low signal-to-noise ratio, which is especially problematic for in vivo imaging, which must contend with light scattering and autofluorescence within tissue. Some of these issues also hamper two-photon imaging, the preferred imaging modality for deep-tissue in vivo imaging.

In contrast to calcium sensors, voltage sensors are targeted to the cell membrane, which is where they are needed. Therefore, "generally you are dealing with a much higher number of sensor molecules per cell for calcium than for voltage [imaging]," Schnitzer explains. In addition, photobleached sensors are replenished less quickly in the membrane than in the cytoplasm, because of slower diffusion in the membrane.

Furthermore, voltage changes, and action potentials in particular, occur on a timescale of milliseconds, in contrast to calcium changes, which last 100 or more milliseconds. Thus the imaging speed has to be much higher for voltage imaging than for calcium imaging. But "if you expose only a fraction of the time, you get a fraction of the photons," says Knöpfel. And low photon counts only worsen the noise problem. This is one of the reasons that Knöpfel prefers mesoscopic imaging, where the larger area being recorded increases the photon count and signal-tonoise ratios.

The fast imaging speeds-in the range of $1 \mathrm{kHz}$ - that are necessary for voltage imaging also pose a problem for two-photon imaging. The laser-scanning approach used in this mode further decreases the window of photon collection per pixel and therefore reduces the photon count even more, in contrast to one-photon wide-field imaging, where each pixel is sampled for the entire time an image frame is acquired. Despite these issues, several of the sensors with voltage-sensitive domains and fluorescent proteins as readout have been imaged with

two-photon microscopy. "ASAP1, the one from Michael Lin's lab, gives the best signal," Cohen says, but the signals are still a long way from being useful in a mouse brain. The opsin-based sensors, however, may not be amenable to two-photon excitation. "It seems to be a principle problem and that has to do with the two-photon cross-section ... because one needs to hit specifically a certain state in the opsin, which is voltage-sensitive and which has that specific wavelength of excitation," speculates Knöpfel.

These challenges in imaging voltage signals are daunting for tool developers in this area. "I think one of the things that makes this field both interesting and challenging is that simply working on the reporters in isolation is rarely sufficient to actually make biological headway," muses Cohen. On the contrary, there is a need for research on the molecular sensors to proceed in parallel with work on instrumentation, optics and computational analytical tools, he says.

A separate issue is that voltage sensors measure relative changes in voltage and

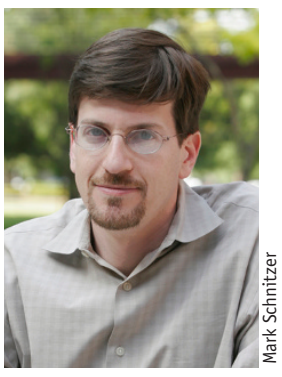

The ability to detect single action potentials in awake mice is not too far away, says Mark

Schnitzer.

$$
\text { any cell depends on }
$$

the amount of reporter, whether it's expression or dye loading, and the illumination intensity and the optics," says Cohen. If a voltage-insensitive fluorescent protein is used at the same time, ratiometric measurements may provide a means to calibrate voltage sensors, but this can also be complicated, as the proteins may photobleach at different rates.

Finally, it is interesting to consider what voltage sensors measure exactly. In conventional electrophysiological recordings, measurements are taken of the voltage difference across the cell membrane. But voltage sensors are embedded in the membrane and exposed to the electrical field on both sides of the membrane and within the membrane. "The electric field profile across the membrane ... 
can actually be a quite complicated function, governed by the distribution of charges on the surfaces of the membrane and the lipid composition of the membrane and its dielectric properties," explains Cohen. Thus, it is not entirely clear what the sensors measure and how these measurements differ from electrophysiological recordings.

\section{A peek into the future}

A possible way to overcome some of the challenges in applying voltage sensors in vivo is to use different imaging modalities. This has not been tried yet, but "there are a whole zoo of structured illumination schemes, there is spinning disc confocal and sheet illumination and many other structured illumination microscopies. They have depth-penetration problems, but they can give some structural information," suggests Cohen. He also thinks that photoacoustic imaging and photothermal imaging could be options to help deal with scattering and photobleaching in vivo.

Furthermore, radically different ways of detecting changes in membrane potential are currently being explored. For instance,
Lloyd Hollenberg and colleagues at the University of Melbourne have explored diamond substrates containing nitrogen vacancies as voltage sensors. They have shown in a theoretical, proof-of-principle study that the magnetic field generated by active neurons grown on the diamond substrate can be imaged via its effect on the fluorescence emitted from nitrogen vacancies under illumination. It might also be conceivable to embed tiny diamond crystals with nitrogen vacancies into the neuronal cell membrane, where the changes in voltage could influence their fluorescence emission.

Regarding genetically encoded sensors, Knöpfel is convinced that there is a lot of room for improvement by tuning these tools. Although he thinks that the voltagesensing domains, being taken straight from nature, are as efficient as possible, engineering aspects such as the coupling between voltage-sensing domains and fluorescent proteins could be improved. "I would expect that finally we will end up with at least ten-times better performance than we have at the moment," projects Knöpfel.
Thus, despite the current difficulties, genetically encoded voltage sensors should have a bright future ahead. And this future might not be too far away, according to Schnitzer, who has looked at the problem from a theoretical point of view. "Even though the sensors today are not at the level at which you could reasonably detect single action potentials in [an] awake mouse, when you run the numbers there is reason to think that we are not that far away."

1. Siegel, M.S. \& Isacoff, E.Y. Neuron 19, 735-741 (1997).

2. Akemann, W. et al. J. Neurophysiol. 108, 23232337 (2012).

3. Jin, L. et al. Neuron 75, 779-785 (2012).

4. St-Pierre, F. et al. Nat. Neurosci. 17, 884-889 (2014).

5. Kralj, J.M. et al. Nat. Methods 9, 90-95 (2012).

6. Hochbaum, D.R. et al. Nat. Methods 11, 825-833 (2014).

7. Flytzanis, N.C. et al. Nat. Commun. 5, 4894 (2014).

8. Gong, Y. et al. Nat. Commun. 5, 3674 (2014).

\section{Nina Vogt is an associate editor for} Nature Methods

(nina.vogt@us.nature.com). 